Tohoku Math. J.

65 (2013), 159-178

\title{
MEROMORPHIC CONTINUATIONS OF LOCAL ZETA FUNCTIONS AND THEIR APPLICATIONS TO OSCILLATING INTEGRALS
}

\author{
TOSHIHISA OKADA AND KIYOSHI TAKEUCHI
}

(Received November 17, 2011, revised July 13, 2012)

\begin{abstract}
We introduce a new method which enables us to calculate the coefficients of the poles of local zeta functions very precisely and prove some explicit formulas. Some vanishing theorems for the candidate poles of local zeta functions will be also given. Moreover we apply our method to oscillating integrals and obtain an explicit formula for the coefficients of their asymptotic expansions.
\end{abstract}

1. Introduction. The theory of local zeta functions is an important subject in many fields of mathematics, such as generalized functions, number theory, prehomogeneous vector spaces (see [12] etc.) and singularity theory. The aim of this paper is to study the coefficients of their poles. Let $f$ be a real-valued real analytic function defined on an open neighborhood $U$ of $0 \in \boldsymbol{R}^{n}$ such that $f(0)=0$ and $\varphi \in C_{0}^{\infty}(U)$ a real-valued test function on $U$. Then the integral

$$
Z_{f}(\varphi)(\lambda)=\int_{\boldsymbol{R}^{n}}|f(x)|^{\lambda} \varphi(x) d x
$$

converges locally uniformly on $\{\lambda \in C ; \Re \lambda>0\}$ and defines a holomorphic function there. Moreover $Z_{f}(\varphi)(\lambda)$ can be extended to a meromorphic function on the whole complex plane $\boldsymbol{C}$ (see [1] and [22] etc.). This meromorphic function $Z_{f}(\varphi)$ of $\lambda$ is called the local zeta function (or the complex power) associated to $f$ and $\varphi$. In 1954 in an invited talk at ICM Amsterdam, I. M. Gelfand raised a famous problem of describing the coefficients of the poles of $Z_{f}(\varphi)$ as distributions on $\boldsymbol{R}^{n}$ (see [6] etc.). About 20 years later, Varchenko [22] proved that there exists a subset $P$ of $\boldsymbol{Q}_{<0}$ in which the poles of $Z_{f}(\varphi)$ are contained (see [1] etc. for the details). After this very fundamental paper [22] many authors studied the poles of local zeta functions. For example, an important progress was made by Denef-Sargos [4] in reducing the set $P$ of candidate poles. However, to the best of our knowledge, there seems to be no paper which calculated the coefficients of these poles explicitly in a general setting. In particular, by the preceding results we cannot see how these coefficients depend on the test function $\varphi$ at all.

In this paper, we introduce a new method which enables us to calculate the coefficients of the poles of local zeta functions as precisely as we want. The key idea is the use of a

2000 Mathematics Subject Classification. Primary 14B05; Secondary 14M25, 14N99, 52B20.

Key words and phrases. Local zeta function, toric variety, oscillating integral. 
meromorphic continuation of the distribution

$$
x_{1+}^{l_{1} \lambda+m_{1}} x_{2+}^{l_{2} \lambda+m_{2}} \cdots x_{n+}^{l_{n} \lambda+m_{n}} \in \mathcal{D}^{\prime}\left(\boldsymbol{R}^{n}\right)
$$

$\left(l_{1}, l_{2}, \ldots, l_{n} \in \boldsymbol{R}_{>0}\right.$ and $\left.m_{1}, m_{2}, \ldots, m_{n} \in \boldsymbol{R}_{+}=\boldsymbol{R}_{\geq 0}\right)$ with respect to the complex parameter $\lambda$, which is different from the one traditionally used in the study of local zeta functions (see [1] etc.). See Section 2 for the detail. This meromorphic continuation enables us to get the information on the poles of local zeta functions much more precisely than before. As is clear from the proofs of our results, by our method we can calculate the coefficients of the poles of $Z_{f}(\varphi)$ very precisely once we construct an embedded resolution of the hypersurface $\{x \in U ; f(x)=0\}$. It would be possible to calculate them as precisely as we want by numerical computations. However, since the general formula (which is evident from our proofs) is more involved, we restrict ourselves here to a generic case where the formulas can be stated neatly. From now on, we assume that the hypersurface $\{x \in U ; f(x)=0\}$ has an isolated singular point at $0 \in U \subset \boldsymbol{R}^{n}$. Let

$$
f(x)=\sum_{\alpha \in Z_{+}^{n}} a_{\alpha} x^{\alpha} \quad\left(a_{\alpha} \in \boldsymbol{R}\right)
$$

be the Taylor expansion of $f$ at $0 \in \boldsymbol{R}^{n}$.

Definition 1.1. (i) Let $\Gamma_{+}(f) \subset \boldsymbol{R}_{+}^{n}:=\boldsymbol{R}_{\geq 0}^{n}$ be the convex hull of $\bigcup_{\alpha: a_{\alpha} \neq 0}(\alpha+$ $\left.\boldsymbol{R}_{+}^{n}\right)$ in $\boldsymbol{R}_{+}^{n}$. We call $\Gamma_{+}(f)$ the Newton polyhedron of $f$.

(ii) For each compact face $\gamma \prec \Gamma_{+}(f)$ of $\Gamma_{+}(f)$ we set

$$
f_{\gamma}(x)=\sum_{\alpha \in \gamma \cap \boldsymbol{Z}_{+}^{n}} a_{\alpha} x^{\alpha} \in \boldsymbol{R}\left[x_{1}, x_{2}, \ldots, x_{n}\right] .
$$

We call $f_{\gamma}(x)$ the $\gamma$-part of $f$.

Note that by the condition $f(0)=0$ we have $0 \notin \Gamma_{+}(f)$. From now on, we assume also that $f$ satisfies the following condition.

DEFINITION 1.2. We say that $f$ is non-degenerate if for any compact face $\gamma \prec \Gamma_{+}(f)$ of $\Gamma_{+}(f)$ we have

$$
\left(\frac{\partial f_{\gamma}}{\partial x_{1}}(x), \frac{\partial f_{\gamma}}{\partial x_{2}}(x), \ldots, \frac{\partial f_{\gamma}}{\partial x_{n}}(x)\right) \neq(0,0, \ldots, 0)
$$

at any point $x$ of $\left\{x \in \boldsymbol{R}^{n} ; x_{1} x_{2} \cdots x_{n} \neq 0, f_{\gamma}(x)=0\right\} \subset(\boldsymbol{R} \backslash\{0\})^{n}$.

Recall that generic (i.e., almost all) functions $f$ having a fixed Newton polyhedron are non-degenerate. For the sake of simplicity, let us assume also that $f$ is convenient: for any $1 \leq i \leq n$ we have $\Gamma_{+}(f) \cap\left\{\alpha=\left(\alpha_{1}, \ldots, \alpha_{n}\right) \in \boldsymbol{R}_{+}^{n} ; \alpha_{j}=0\right.$ for $j \neq i$ and $\left.\alpha_{i}>0\right\} \neq \emptyset$. Then by the results of Varchenko [22] (see also [1] and [11]) we can describe the candidate poles of $Z_{f}(\varphi)$ in terms of $\Gamma_{+}(f)$ as follows. 
DEFINITION 1.3 (see [1], [5] and [11] etc.).

(i) A family $\Sigma=\{\sigma\}$ of rational convex polyhedral cones $\sigma$ in $\boldsymbol{R}^{n}$ is called a fan if it satisfies the conditions: (a) If a cone $\sigma$ is an element of $\Sigma$, all its faces are also so. (b) If $\sigma, \tau \in \Sigma$, then $\sigma \cap \tau$ is a common face of $\sigma$ and $\tau$.

(ii) For a rational convex polyhedral cone $\sigma$ in $\boldsymbol{R}^{n}$ the set $\left\{a^{1}(\sigma), a^{2}(\sigma), \ldots, a^{k}(\sigma)\right\}$ of the (non-zero) primitive vectors $a^{i}(\sigma) \in \partial \sigma \cap\left(\boldsymbol{Z}^{n} \backslash\{0\}\right)$ on its edges (i.e., 1-dimensional faces) is called the 1-skeleton of $\sigma$.

(iii) A fan $\Sigma=\{\sigma\}$ in $\boldsymbol{R}^{n}$ is called simplicial (resp. smooth) if for any cone $\sigma \in \Sigma$ its 1-skeleton forms a part of a basis of the vector space $\boldsymbol{R}^{n}$ (resp. the lattice $\boldsymbol{Z}^{n}$ ).

For a face $\gamma \prec \Gamma_{+}(f)$ of $\Gamma_{+}(f)$, let $\sigma(\gamma) \subset \boldsymbol{R}_{+}^{n}$ be the convex polyhedral cone generated by the inner conormal vectors of the $(n-1)$-dimensional faces $\gamma^{\prime} \prec \Gamma_{+}(f)$ such that $\gamma \prec \gamma^{\prime}$ over $\boldsymbol{R}_{+}$. We can easily see that $\operatorname{dim} \sigma(\gamma)=n-\operatorname{dim} \gamma$. It is well known that the family $\{\sigma(\gamma)\}_{\gamma<\Gamma_{+}(f)}$ of cones is a fan in $\boldsymbol{R}^{n}$ satisfying the condition $\boldsymbol{R}_{+}^{n}=\bigcup_{\gamma \prec \Gamma_{+}(f)} \sigma(\gamma)$ (see [1], [5] and [11] etc.).

DEFinition 1.4 (see [1], [5] and [11] etc.). We set $\Sigma_{0}=\{\sigma(\gamma)\}_{\gamma \prec \Gamma_{+}(f)}$ and call it the dual fan of $\Gamma_{+}(f)$.

Let $\Sigma$ be a smooth subdivision of $\Sigma_{0}$. Note that $\Sigma=\{\sigma\}$ is a family of rational simplicial polyhedral cones in $\boldsymbol{R}_{+}^{n}$ such that $\boldsymbol{R}_{+}^{n}=\bigcup_{\sigma \in \Sigma} \sigma$. For a cone $\sigma$ in $\Sigma$ let $\left\{a^{1}(\sigma), a^{2}(\sigma), \ldots\right.$, $\left.a^{\operatorname{dim} \sigma}(\sigma)\right\} \subset \partial \sigma \cap\left(\boldsymbol{Z}^{n} \backslash\{0\}\right)$ be the 1-skeleton of $\sigma$. Recall that by the smoothness of $\Sigma$ there exist exactly $\operatorname{dim} \sigma$ edges on $\sigma$ and the (non-zero) primitive vectors $a^{1}(\sigma), a^{2}(\sigma), \ldots$, $a^{\operatorname{dim} \sigma}(\sigma)$ form a part of a basis of the lattice $\boldsymbol{Z}^{n}$. For each $n$-dimensional cone $\sigma \in \Sigma$ we fix the ordering of its 1-skeleton $\left\{a^{1}(\sigma), a^{2}(\sigma), \ldots, a^{n}(\sigma)\right\}$ so that the determinant of the invertible matrix

$$
A(\sigma)=\left\{a^{i}(\sigma)_{j}\right\}_{i, j=1}^{n} \in G L_{n}(\boldsymbol{Z})
$$

is 1 . For a cone $\sigma \in \Sigma$ and $1 \leq i \leq \operatorname{dim} \sigma$ we set

$$
l\left(a^{i}(\sigma)\right)=\min _{\alpha \in \Gamma_{+}(f)}\left\langle a^{i}(\sigma), \alpha\right\rangle \in \boldsymbol{Z}_{+}
$$

and

$$
\left|a^{i}(\sigma)\right|=\sum_{j=1}^{n} a^{i}(\sigma)_{j} \in \boldsymbol{Z}_{>0} .
$$

For $0 \leq k \leq n$ let $\Sigma^{(k)} \subset \Sigma$ be the subset of $\Sigma$ consisting of $k$-dimensional cones.

DEFINITION 1.5 (see [1] and [22] etc.). Let $P \subset \boldsymbol{Q}_{<0}$ be the union of the following subsets of $\boldsymbol{Q}_{<0}$ :

$$
\begin{gathered}
\{-1,-2,-3, \ldots\}, \\
\left\{-\frac{\left|a^{1}(\sigma)\right|}{l\left(a^{1}(\sigma)\right)},-\frac{\left|a^{1}(\sigma)\right|+1}{l\left(a^{1}(\sigma)\right)}, \ldots\right\} \quad\left(\sigma \in \Sigma^{(1)} \text { such that } l\left(a^{1}(\sigma)\right)>0\right) .
\end{gathered}
$$


By the fundamental result of [22], the poles of $Z_{f}(\varphi)$ are contained in $P$. We call an element of $P$ a candidate pole of $Z_{f}(\varphi)$. Let us order the candidate poles of $Z_{f}(\varphi)$ as

$$
P=\left\{-\lambda_{1}>-\lambda_{2}>-\lambda_{3}>\cdots\right\} \quad\left(\lambda_{j} \in Q_{>0}\right) .
$$

Definition 1.6. (i) Let $\sigma \in \Sigma$. For $1 \leq i \leq \operatorname{dim} \sigma$ such that $l\left(a^{i}(\sigma)\right)>0(\Longleftrightarrow$ $\left.a^{i}(\sigma) \in \boldsymbol{R}_{>0}^{n}\right)$ we set

$$
K^{i}(\sigma)=\left\{\frac{\left|a^{i}(\sigma)\right|}{l\left(a^{i}(\sigma)\right)}, \frac{\left|a^{i}(\sigma)\right|+1}{l\left(a^{i}(\sigma)\right)}, \ldots\right\} \subset \boldsymbol{Q}_{>0} .
$$

(ii) For the $j$-th candidate pole $-\lambda_{j} \in P$ of $Z_{f}(\varphi)$ and $0 \leq k \leq n$ we define a subset $\Sigma_{j}^{(k)}$ of $\Sigma^{(k)}$ by

(1.13) $\Sigma_{j}^{(k)}=\left\{\sigma \in \Sigma^{(k)} ; l\left(a^{i}(\sigma)\right)>0 \quad\right.$ and $\quad \lambda_{j} \in K^{i}(\sigma)$ for any $\left.1 \leq i \leq k=\operatorname{dim} \sigma\right\}$.

(iii) For $\sigma \in \Sigma_{j}^{(k)}$ and $1 \leq i \leq k$ we define a non-negative integer $\nu(\sigma)_{i} \in \boldsymbol{Z}_{+}$by

$$
\lambda_{j}=\frac{\left|a^{i}(\sigma)\right|+v(\sigma)_{i}}{l\left(a^{i}(\sigma)\right)} .
$$

For the sake of simplicity, in this introduction we assume that $-\lambda_{j} \in P$ is not an integer. Then it is well known that the order of the pole of $Z_{f}(\varphi)$ at $\lambda=-\lambda_{j}$ is less than or equal to

$$
k_{j}:=\max \left\{0 \leq k \leq n ; \Sigma_{j}^{(k)} \neq \emptyset\right\} \in \boldsymbol{Z}_{+} .
$$

Let

$$
\frac{a_{j, k_{j}}(\varphi)}{\left(\lambda+\lambda_{j}\right)^{k_{j}}}+\cdots+\frac{a_{j, 2}(\varphi)}{\left(\lambda+\lambda_{j}\right)^{2}}+\frac{a_{j, 1}(\varphi)}{\left(\lambda+\lambda_{j}\right)}+\cdots \quad\left(a_{j, k}(\varphi) \in \boldsymbol{R}\right)
$$

be the Laurent expansion of $Z_{f}(\varphi)$ at $\lambda=-\lambda_{j}$. Then we obtain the following vanishing theorem which generalizes Jacobs [10, Theorem 4.23].

THEOREM 1.7. Let $1 \leq k \leq k_{j}$. Assume that for any $\sigma \in \Sigma_{j}^{(k)}$ there exists $1 \leq i \leq$ $k=\operatorname{dim} \sigma$ such that $v(\sigma)_{i}$ is odd. Then we have $a_{j, k}(\varphi)=\cdots=a_{j, k_{j}}(\varphi)=0$.

In order to state our another vanishing theorem, let

$$
\varphi(x)=\sum_{\alpha \in \boldsymbol{Z}_{+}^{n}} c_{\alpha} x^{\alpha} \quad\left(c_{\alpha}=\frac{\partial_{x}^{\alpha} \varphi(0)}{\alpha !} \in \boldsymbol{R}\right)
$$

be the (formal) Taylor expansion of the test function $\varphi$ at $0 \in U \subset \boldsymbol{R}^{n}$.

THEOREM 1.8. For $1 \leq k \leq k_{j}$ assume that $\left\{\alpha \in Z_{+}^{n} ; c_{\alpha} \neq 0\right\} \cap \Delta_{j, k}=\emptyset$, where $\Delta_{j, k}$ is a certain compact subset of $\boldsymbol{R}_{+}^{n}$ (to be defined in Definition 3.4). Then we have $a_{j, k}(\varphi)=\cdots=a_{j, k_{j}}(\varphi)=0$.

Moreover the coefficients $a_{j, n}(\varphi)$ of the deepest poles $\lambda=-\lambda_{j} \in P$ can be explicitly described by the following formula. For $\sigma \in \Sigma_{j}^{(n)}, 1 \leq i \leq n$ and $\alpha \in Z_{+}^{n}$ we define an 
integer $\mu(\sigma, \alpha)_{i}$ by

$$
\mu(\sigma, \alpha)_{i}=v(\sigma)_{i}-\left\langle a^{i}(\sigma), \alpha\right\rangle \in \boldsymbol{Z} .
$$

We also define a function $f_{\sigma}$ on a neighborhood of $0 \in \boldsymbol{R}_{y}^{n}$ satisfying $f_{\sigma}(0) \neq 0$ by

$$
f_{\sigma}(y)=\sum_{\alpha \in Z_{+}^{n}} a_{\alpha} \prod_{i=1}^{n} y_{i}^{\left\langle a^{i}(\sigma), \alpha\right\rangle-l\left(a^{i}(\sigma)\right)}
$$

by using the Taylor expansion $f(x)=\sum_{\alpha \in Z_{+}^{n}} a_{\alpha} x^{\alpha}$ of $f$.

THEOREM 1.9. Assume that $k_{j}=n$. Then $a_{j, n}(\varphi)$ is given by

$$
a_{j, n}(\varphi)=\sum_{\alpha \in \Delta_{j, n}}\left\{\sum_{\sigma \in \Sigma_{j}^{(n)}}\left(\prod_{i=1}^{n} \frac{1+(-1)^{\nu(\sigma)_{i}}}{l\left(a^{i}(\sigma)\right) \cdot \mu(\sigma, \alpha)_{i} !}\right) \partial_{y}^{\mu(\sigma, \alpha)}\left|f_{\sigma}\right|^{-\lambda_{j}}(0)\right\} \frac{\partial_{x}^{\alpha} \varphi(0)}{\alpha !}
$$

where we set

$$
\partial_{y}^{\mu(\sigma, \alpha)}=\frac{\partial^{\mu(\sigma, \alpha)_{1}+\cdots+\mu(\sigma, \alpha)_{n}}}{\partial y_{1}^{\mu(\sigma, \alpha)_{1}} \cdots \partial y_{n}^{\mu(\sigma, \alpha)_{n}}}
$$

and $\left(\partial^{\mu} / \partial y_{i}^{\mu}\right)(\cdot)=0$ if $\mu<0$.

Our results on the poles of the local zeta function $Z_{f}(\varphi)$ also have some applications to the oscillating integral $I_{f}(\varphi)(t)(t \in \boldsymbol{R})$ defined by

$$
I_{f}(\varphi)(t)=\int_{\boldsymbol{R}^{n}} e^{i t f(x)} \varphi(x) d x .
$$

By the fundamental results of Varchenko [22] (see also [1] for the detail), as $t \rightarrow+\infty$ the oscillating integral $I_{f}(\varphi)(t)$ has an asymptotic expansion of the form

$$
I_{f}(\varphi)(t) \sim \sum_{j=1}^{\infty} \sum_{k=1}^{k_{j}} c_{j, k}(\varphi) t^{-\lambda_{j}}(\log t)^{k-1},
$$

where $c_{j, k}(\varphi)$ are some complex numbers. Despite the important contributions by many mathematicians (see for example [1], [3], [8] and [20] etc.), only little is known about the coefficients $c_{j, k}(\varphi)$ of this asymptotic expansion. Here we can prove the following general vanishing theorem.

THEOREM 1.10. In the situation as above, let $1 \leq k \leq k_{j}$ and assume that $\{\alpha \in$ $\left.\boldsymbol{Z}_{+}^{n} ; c_{\alpha} \neq 0\right\} \cap \Delta_{j, k}=\emptyset$. Then we have $c_{j, k}(\varphi)=\cdots=c_{j, k_{j}}(\varphi)=0$.

Moreover by Theorem 1.9 we obtain also an explicit formula for the coefficients $c_{j, n}(\varphi)$ of $t^{-\lambda_{j}}(\log t)^{n-1}$ in the asymptotic expansion of $I_{f}(\varphi)$. See Section 5 for the detail. Note that recently by the second author the method of toric modifications used in this paper was applied also to various problems of mathematics in [13], [14], [15] and [21] etc. Finally let us mention that the method introduced in this paper would have some applications also in the study of $p$-adic local zeta functions (see [9] etc.). It would be a very interesting subject to study the twisted monodromy conjecture (see [16] for a review on this conjecture) by this method. 
2. Meromorphic continuations of distributions. In this section, as a preparation we prove some basic results on the meromorphic continuations of the distributions $x_{1+}^{l_{1} \lambda+m_{1}} x_{2+}^{l_{2} \lambda+m_{2}} \cdots x_{n+}^{l_{n} \lambda+m_{n}}\left(l_{i} \in \boldsymbol{R}_{>0}, m_{i} \in \boldsymbol{R}_{+}=\boldsymbol{R}_{\geq 0}\right)$ with respect to the complex parameter $\lambda$. In Sections 3 and 4 these results will be used effectively to study the poles of local zeta functions. First, let us recall the classical result in the 1-dimensional case (see GelfandShilov [6] etc.). Let $l \in \boldsymbol{R}_{>0}$ be a positive real number and $m \in \boldsymbol{R}_{+}$. Then for $\varphi \in C_{0}^{\infty}(\boldsymbol{R})$ the integral

$$
F_{+}(\varphi)(\lambda)=\int_{-\infty}^{+\infty} x_{+}^{l \lambda+m} \varphi(x) d x=\int_{0}^{+\infty} x^{l \lambda+m} \varphi(x) d x
$$

converges locally uniformly on $\{\lambda \in \boldsymbol{C} ; \mathfrak{R} \lambda>-(m+1) / l\}$ and defines a holomorphic function there. In other words, if $\Re \lambda>-(m+1) / l$ the map $\varphi \mapsto F_{+}(\varphi)(\lambda) \in \boldsymbol{R}$ defines a distribution $x_{+}^{l \lambda+m} \in \mathcal{D}^{\prime}(\boldsymbol{R})$ on $\boldsymbol{R}$. Let us fix a test function $\varphi \in C_{0}^{\infty}(\boldsymbol{R})$. Following the methods in Gelfand-Shilov [8] we shall extend $F_{+}(\varphi)$ to a meromorphic function on the whole complex plane $\boldsymbol{C}$ as follows. First take a sufficiently large integer $N \gg 0$. Then for any $\lambda \in \boldsymbol{C}$ such that $\Re \lambda>-(m+1) / l$ we have

$$
\begin{aligned}
F_{+}(\varphi)(\lambda)= & \int_{0}^{+\infty} x^{l \lambda+m} \varphi(x) d x \\
= & \int_{0}^{1} x^{l \lambda+m}\left[\varphi(x)-\sum_{r=1}^{N} \varphi^{(r-1)}(0) \frac{x^{r-1}}{(r-1) !}\right] d x \\
& +\int_{1}^{+\infty} x^{l \lambda+m} \varphi(x) d x+\sum_{r=1}^{N} \frac{\varphi^{(r-1)}(0)}{(r-1) !(l \lambda+m+r)} \\
= & \int_{0}^{1} x^{l \lambda+m} d x \int_{0}^{x} \frac{\varphi^{(N)}(t)}{(N-1) !}(x-t)^{N-1} d t+\int_{1}^{+\infty} x^{l \lambda+m} \varphi(x) d x \\
& +\sum_{r=1}^{N} \frac{\varphi^{(r-1)}(0)}{(r-1) ! \times l\{\lambda+(m+r) / l\}} \\
= & \int_{0}^{1} g_{N}(\lambda, t) \varphi^{(N)}(t) d t+\int_{1}^{+\infty} t^{l \lambda+m} \varphi(t) d t \\
& +\sum_{r=1}^{N} \frac{1}{(r-1) ! \times l\{\lambda+(m+r) / l\}}\left\langle(-1)^{r-1} \delta^{(r-1)}, \varphi\right\rangle,
\end{aligned}
$$

where $\delta \in \mathcal{D}^{\prime}(\boldsymbol{R})$ is Dirac's delta function and we set

$$
g_{N}(\lambda, t)=\frac{1}{(N-1) !} \int_{t}^{1} x^{l \lambda+m}(x-t)^{N-1} d x
$$

for $0<t \leq 1$. The function $g_{N}(\lambda, t)$ satisfies the following nice properties.

LEMMA 2.1. (i) For any $0<t \leq 1, g_{N}(\lambda, t)$ is an entire function of $\lambda$.

(ii) If $\Re \lambda>-(m+N+1) / l$ then the function $g_{N}(\lambda, t)$ of $t$ is integrable on $(0,1]$. 
By this lemma we see that the integral

$$
\int_{0}^{1} g_{N}(\lambda, t) \varphi^{(N)}(t) d t
$$

converges locally uniformly on $\{\lambda \in \boldsymbol{C} ; \Re \lambda>-(m+N+1) / l\}$ and defines a holomorphic function there. Since the integral $\int_{1}^{+\infty} t^{l \lambda+m} \varphi(t) d t$ is an entire function of $\lambda$, the function $F_{+}(\varphi)$ is extended to a meromorphic function on $\{\lambda \in C ; \Re \lambda>-(m+N+1) / l\}$ with simple poles at $\lambda=-(m+r) / l(r=1,2, \ldots, N)$. Moreover the residue of $F_{+}(\varphi)$ at $\lambda=-(m+r) / l$ is given by

$$
\operatorname{Res}\left(F_{+}(\varphi) ;-\frac{m+r}{l}\right)=\frac{1}{(r-1) ! \times l}\left\langle(-1)^{r-1} \delta^{(r-1)}, \varphi\right\rangle .
$$

Similarly set

$$
F_{-}(\varphi)(\lambda)=\int_{-\infty}^{+\infty} x_{-}^{l \lambda+m} \varphi(x) d x=\int_{-\infty}^{0}|x|^{l \lambda+m} \varphi(x) d x .
$$

Then $F_{-}(\varphi)(\lambda)$ can be also extended to a meromorphic function on the whole complex plane $C$ with simple poles at $\lambda=-(m+r) / l(r=1,2, \ldots)$ and we have

$$
\operatorname{Res}\left(F_{-}(\varphi) ;-\frac{m+r}{l}\right)=\frac{1}{(r-1) ! \times l}\left\langle\delta^{(r-1)}, \varphi\right\rangle .
$$

This basic result in the 1-dimensional case can be generalized to higher-dimensional cases as follows. Let $\varphi \in C_{0}^{\infty}\left(\boldsymbol{R}^{n}\right)$ be a test function on $\boldsymbol{R}^{n}$. For positive real numbers $l_{1}, l_{2}, \ldots, l_{n} \in$ $\boldsymbol{R}_{>0}$ and $m_{1}, m_{2}, \ldots, m_{n} \in \boldsymbol{R}_{+}$we set

$$
G(\varphi)(\lambda)=\int_{\boldsymbol{R}^{n}} x_{1+}^{l_{1} \lambda+m_{1}} x_{2+}^{l_{2} \lambda+m_{2}} \cdots x_{n+}^{l_{n} \lambda+m_{n}} \varphi(x) d x
$$

and

$$
L=-\min \left\{\frac{m_{1}+1}{l_{1}}, \frac{m_{2}+1}{l_{2}}, \ldots, \frac{m_{n}+1}{l_{n}}\right\} .
$$

Then this integral converges locally uniformly on $\{\lambda \in C ; \Re \lambda>L\}$ and defines a holomorphic function there. By using the tensor product $\otimes$ of distributions we can rewrite $G(\varphi)(\lambda)$ as

$$
G(\varphi)(\lambda)=\left\langle x_{1+}^{l_{1} \lambda+m_{1}} \otimes x_{2+}^{l_{2} \lambda+m_{2}} \otimes \cdots \otimes x_{n+}^{l_{n} \lambda+m_{n}}, \varphi\right\rangle .
$$

Let $N \gg 0$ be a sufficiently large integer. Then for $\lambda \in C$ such that $\Re \lambda>L$ we have the following equalities in the space $\mathcal{D}^{\prime}(\boldsymbol{R})$ of 1-dimensional distributions.

$$
\begin{aligned}
x_{i+}^{l_{i} \lambda+m_{i}}= & G_{i, N}(\lambda) \\
& +\sum_{r=1}^{N} \frac{(-1)^{r-1}}{(r-1) ! \times l_{i}\left\{\lambda+\left(m_{i}+r\right) / l_{i}\right\}} \delta^{(r-1)}\left(x_{i}\right) \quad(i=1,2, \ldots, n),
\end{aligned}
$$


where $G_{i, N}(\lambda) \in \mathcal{D}^{\prime}(\boldsymbol{R})$ is a 1-dimensional distribution of the form

$$
\left\langle G_{i, N}(\lambda), \phi\right\rangle=\int_{0}^{1} g_{i, N}(\lambda, t) \phi^{(N)}(t) d t+\int_{1}^{+\infty} t^{l_{i} \lambda+m_{i}} \phi(t) d t \quad\left(\phi \in C_{0}^{\infty}(\boldsymbol{R})\right) .
$$

Here $g_{i, N}(\lambda, t)$ is an integrable function of $t \in(0,1]$ for $\lambda \in C$ such that $\Re \lambda>-\left(m_{i}+N+\right.$ 1) $/ l_{i}$. Putting these new expressions of the 1-dimensional distributions $x_{i+}^{l_{i} \lambda+m_{i}}$ into (2.10) we see that $G(\varphi)(\lambda)$ is extended to a meromorphic function on $\left\{\lambda \in C ; \Re \lambda>L_{N}\right\}$, where we set

$$
L_{N}=-\min \left\{\frac{m_{1}+N+1}{l_{1}}, \frac{m_{2}+N+1}{l_{2}}, \ldots, \frac{m_{n}+N+1}{l_{n}}\right\} .
$$

Since the integer $N \gg 0$ can be taken as large as possible, $G(\varphi)(\lambda)$ is meromorphically continued to the whole complex plane $\boldsymbol{C}$. Moreover the poles of this meromorphic function $G(\varphi)(\lambda)$ are contained in a discrete subset $P$ of $C$ defined by

$$
P=\bigcup_{1 \leq i \leq n}\left\{-\frac{m_{i}+1}{l_{i}},-\frac{m_{i}+2}{l_{i}},-\frac{m_{i}+3}{l_{i}}, \ldots\right\} \subset \boldsymbol{R}_{<0} \subset \boldsymbol{C} .
$$

An element of $P$ is called a candidate pole of $G(\varphi)$. Let us rewrite this set $P$ as

$$
P=\left\{-\lambda_{1}>-\lambda_{2}>-\lambda_{3}>\cdots\right\} \quad\left(\lambda_{j} \in \boldsymbol{R}_{>0}\right) .
$$

For each candidate pole $-\lambda_{j} \in P$ of $G(\varphi)$ we define a subset $S_{j}$ of $\{1,2, \ldots, n\}$ by

$$
S_{j}=\left\{1 \leq i \leq n ;{ }^{\exists} r \in \boldsymbol{Z}_{>0} \text { such that } \frac{m_{i}+r}{l_{i}}=\lambda_{j}\right\}
$$

and set $k_{j}=\sharp S_{j}$. Then we can easily see that the order of the pole of $G(\varphi)$ at $\lambda=-\lambda_{j}$ is less than or equal to $k_{j}$. For a candidate pole $-\lambda_{j} \in P$ of $G(\varphi)$ let

$$
\frac{a_{j, k_{j}}}{\left(\lambda+\lambda_{j}\right)^{k_{j}}}+\cdots+\frac{a_{j, 2}}{\left(\lambda+\lambda_{j}\right)^{2}}+\frac{a_{j, 1}}{\left(\lambda+\lambda_{j}\right)}+\cdots \quad\left(a_{j, k} \in \boldsymbol{R}\right)
$$

be the Laurent expansion of $G(\varphi)$ at $\lambda=-\lambda_{j}$. For each $i \in S_{j} \subset\{1,2, \ldots, n\}$ we define a non-negative integer $v_{i} \in \boldsymbol{Z}_{+}=\boldsymbol{Z}_{\geq 0}$ by the formula

$$
\frac{m_{i}+1+v_{i}}{l_{i}}=\lambda_{j} .
$$

Proposition 2.2. Let $1 \leq k \leq k_{j}$. Then the coefficient $a_{j, k}$ of $1 /\left(\lambda+\lambda_{j}\right)^{k}$ in the Laurent expansion of $G(\varphi)(\lambda)$ at $\lambda=-\lambda_{j}$ is written as

$$
a_{j, k}=\sum_{S \subset S_{j}: \sharp S \geq k} \frac{1}{(\sharp S-k) !}\left\{\frac{\partial^{\sharp S-k}}{\partial \lambda \sharp S-k} \rho_{S}(\lambda)\right\}_{\lambda=-\lambda_{j}},
$$

where for each subset $S \subset S_{j}$ of $S_{j}$ such that $\sharp S \geq k$ the function $\rho_{S}(\lambda)$ is holomorphic at $\lambda=-\lambda_{j}$ and written as follows:

For the sake of simplicity, assume that $S=\{1,2, \ldots, p\}$ for some $p \geq k$. Then we have

$$
\rho_{S}(\lambda)=\left(\prod_{i=1}^{p} \frac{1}{l_{i} \cdot v_{i} !}\right) \times \int_{\left\{x \in \boldsymbol{R}^{n} ; x_{1}=\cdots=x_{p}=0\right\}} \prod_{i=p+1}^{n} g_{i}\left(\lambda, x_{i}\right)
$$




$$
\times\left\{\frac{\partial^{v_{1}+\cdots+v_{p}}}{\partial x_{1}^{\nu_{1}} \cdots \partial x_{p}^{v_{p}}} \varphi(x)\right\}_{x_{1}=\cdots=x_{p}=0} d x_{p+1} \cdots d x_{n},
$$

where $g_{i}(\lambda, \cdot)(i=p+1, \ldots, n)$ are 1-dimensional integrable functions with holomorphic parameter $\lambda$ at $\lambda=-\lambda_{j}$.

When $k_{j}=\sharp S_{j}=n$ we have the following very simple expression of $a_{j, n}$.

Proposition 2.3. If $k_{j}=\sharp S_{j}=n$, we have

$$
a_{j, n}=\left(\prod_{i=1}^{n} \frac{1}{l_{i} \cdot v_{i} !}\right) \frac{\partial^{v_{1}+\cdots+v_{n}}}{\partial x_{1}^{\nu_{1}} \cdots \partial x_{n}^{v_{n}}} \varphi(0) .
$$

Similarly let us set

$$
H(\varphi)(\lambda)=\int_{\boldsymbol{R}^{n}}\left|x_{1}\right|^{l_{1} \lambda+m_{1}}\left|x_{2}\right|^{l_{2} \lambda+m_{2}} \cdots\left|x_{n}\right|^{l_{n} \lambda+m_{n}} \varphi(x) d x .
$$

Then $H(\varphi)$ can be also extended to a meromorphic function on $\boldsymbol{C}$ whose poles are contained in the set $P \subset \boldsymbol{R}_{<0}$. Moreover the order of the pole of $H(\varphi)$ at $\lambda=-\lambda_{j} \in P$ is less than or equal to $k_{j}$. For a candidate pole $-\lambda_{j} \in P$ of $H(\varphi)$, let

$$
\frac{b_{j, k_{j}}}{\left(\lambda+\lambda_{j}\right)^{k_{j}}}+\cdots+\frac{b_{j, 2}}{\left(\lambda+\lambda_{j}\right)^{2}}+\frac{b_{j, 1}}{\left(\lambda+\lambda_{j}\right)}+\cdots \quad\left(b_{j, k} \in \boldsymbol{R}\right)
$$

be the Laurent expansion of $H(\varphi)$ at $\lambda=-\lambda_{j}$.

Proposition 2.4. Let $1 \leq k \leq k_{j}$. Then the coefficient $b_{j, k}$ of $1 /\left(\lambda+\lambda_{j}\right)^{k}$ in the Laurent expansion of $H(\varphi)(\lambda)$ at $\lambda=-\lambda_{j}$ is written as

$$
b_{j, k}=\sum_{S \subset S_{j}: \sharp S \geq k} \frac{1}{(\sharp S-k) !}\left\{\frac{\partial^{\sharp S-k}}{\partial \lambda \sharp S-k} \tau_{S}(\lambda)\right\}_{\lambda=-\lambda_{j}},
$$

where, for each subset $S \subset S_{j}$ of $S_{j}$ such that $\sharp S \geq k$, the function $\tau_{S}(\lambda)$ is holomorphic at $\lambda=-\lambda_{j}$ and written as follows:

For the sake of simplicity, assume that $S=\{1,2, \ldots, p\}$ for some $p \geq k$. Then we have

$$
\begin{aligned}
\tau_{S}(\lambda)= & \left(\prod_{i=1}^{p} \frac{1+(-1)^{v_{i}}}{l_{i} \cdot v_{i} !}\right) \times \int_{\left\{x \in \boldsymbol{R}^{n} ; x_{1}=\cdots=x_{p}=0\right\}} \prod_{i=p+1}^{n} g_{i}^{\prime}\left(\lambda, x_{i}\right) \\
& \times\left\{\frac{\partial^{\nu_{1}+\cdots+v_{p}}}{\partial x_{1}^{\nu_{1}} \cdots \partial x_{p}^{v_{p}}} \varphi(x)\right\}_{x_{1}=\cdots=x_{p}=0} d x_{p+1} \cdots d x_{n},
\end{aligned}
$$

where $g_{i}^{\prime}(\lambda, \cdot)(i=p+1, \ldots, n)$ are 1-dimensional integrable functions as in Proposition 2.2 .

As a special case of this proposition, we obtain the following.

COROLlary 2.5. Let $1 \leq k \leq k_{j}$. Assume that for any $S \subset S_{j}$ with $\sharp S=k$ there exists $i \in S$ such that $v_{i}$ is odd. Then we have $b_{j, k}=\cdots=b_{j, k_{j}}=0$.

If $k_{j}=\sharp S_{j}=n$, we can also obtain the following explicit expression of $b_{j, n}$. 
Proposition 2.6. If $k_{j}=\sharp S_{j}=n$, we have

$$
b_{j, n}=\left(\prod_{i=1}^{n} \frac{1+(-1)^{v_{i}}}{l_{i} \cdot v_{i} !}\right) \frac{\partial^{v_{1}+\cdots+v_{n}}}{\partial x_{1}^{\nu_{1}} \cdots \partial x_{n}^{v_{n}}} \varphi(0) \text {. }
$$

REMARK 2.7. Combining our idea above with [2, Petit lemme on p.136] (instead of the meromorphic continuations of Gelfand-Shilov [6] etc.) we can prove some results similar to the ones in this section. We thank the referee for pointing out this alternative approach.

3. Vanishing theorems for the poles of local zeta functions. Let $f$ be a real-valued real analytic function defined on an open neighborhood $U$ of $0 \in \boldsymbol{R}^{n}$ such that $f(0)=0$. Then for any real-valued test function $\varphi \in C_{0}^{\infty}(U)$, the integral

$$
Z_{f}(\varphi)(\lambda)=\int_{\boldsymbol{R}^{n}}|f(x)|^{\lambda} \varphi(x) d x
$$

converges locally uniformly on $\{\lambda \in C ; \Re \lambda>0\}$ and defines a holomorphic function there. Moreover it is well known that $Z_{f}(\varphi)(\lambda)$ can be extended to a meromorphic function defined on the whole complex plane $\boldsymbol{C}$ (see [1], [11] and [22] etc.). In this section, assuming that the real hypersurface $\{x \in U ; f(x)=0\}$ has an isolated singular point at $0 \in U \subset \boldsymbol{R}^{n}$, we prove some general vanishing theorems on the poles of the local zeta function $Z_{f}(\varphi)(\lambda)$. As in Section 1 we assume also that $f$ is convenient and non-degenerate. Let $\Sigma$ be a smooth subdivision of the dual fan $\Sigma_{0}$ of $\Gamma_{+}(f)$. Then by the general theory of toric varieties as in Fulton [5], to the smooth fan $\Sigma$ in $\boldsymbol{R}^{n}$ such that $\boldsymbol{R}_{+}^{n}=\bigcup_{\sigma \in \Sigma} \sigma$ we can naturally associate an $n$-dimensional real analytic manifold $X_{\Sigma}$ and a proper morphism

$$
\pi: X_{\Sigma} \longrightarrow \boldsymbol{R}^{n}
$$

of real analytic manifolds. Here we simply recall that for any $\sigma \in \Sigma$ there exists a subset $O_{\sigma} \simeq(\boldsymbol{R} \backslash\{0\})^{n-\operatorname{dim} \sigma}$ of $X_{\Sigma}$ such that $X_{\Sigma}=\bigsqcup_{\sigma \in \Sigma} O_{\sigma}$. For each $n$-dimensional cone $\sigma$ in $\Sigma$ the open subset $\boldsymbol{R}^{n}(\sigma):=\bigsqcup_{\tau \prec \sigma} O_{\tau}$ of $X_{\Sigma}$ is isomorphic to $\boldsymbol{R}^{n}$ and $O_{\{0\}} \subset \boldsymbol{R}^{n}(\sigma)$ (resp. $\left.O_{\sigma} \subset \boldsymbol{R}^{n}(\sigma)\right)$ corresponds to the standard open dense real torus $(\boldsymbol{R} \backslash\{0\})^{n}$ (resp. the origin 0 ) in $\boldsymbol{R}^{n}$. It follows immediately from this construction that if $\sigma \neq \tau$ are $n$-dimensional cones in $\Sigma$ the origins of $\boldsymbol{R}^{n}(\sigma) \simeq \boldsymbol{R}^{n}$ and $\boldsymbol{R}^{n}(\tau) \simeq \boldsymbol{R}^{n}$ are disjoint in $X_{\Sigma}$. By the convenience of $f$ we can construct the smooth fan $\Sigma$ without subdividing the cones of $\Sigma_{0}$ contained in $\partial \boldsymbol{R}_{+}^{n}$ so that $\pi$ induces an isomorphism

$$
X_{\Sigma} \backslash \pi^{-1}(0) \stackrel{\sim}{\longrightarrow} \boldsymbol{R}^{n} \backslash\{0\} .
$$

Moreover by the non-degeneracy of $f$, the pull-back $f \circ \pi: X_{\Sigma} \longrightarrow \boldsymbol{R}$ of $f$ to $X_{\Sigma}$ defines a hypersurface $\{f \circ \pi=0\}$ in $X_{\Sigma}$ having only normal crossing singularities in $\pi^{-1}(U)$ (see [1], [11], [18] and [22] etc.). Indeed, on each affine open subset $\boldsymbol{R}^{n}(\sigma) \simeq \boldsymbol{R}^{n}$ of $X_{\Sigma}$ the morphism $\pi: X_{\Sigma} \longrightarrow \boldsymbol{R}^{n}$ and the function $f \circ \pi$ can be very explicitly written as follows. First, note that by the smoothness of $\Sigma$ any cone $\sigma \in \Sigma$ is simplicial and hence there exist exactly $\operatorname{dim} \sigma$ edges on it. For a cone $\sigma \in \Sigma$ let $\left\{a^{1}(\sigma), a^{2}(\sigma), \ldots, a^{\operatorname{dim} \sigma}(\sigma)\right\}$ be the 1skeleton of $\sigma$. For each $n$-dimensional cone $\sigma \in \Sigma$ we fix the ordering of its 1-skeleton 
$\left\{a^{1}(\sigma), a^{2}(\sigma), \ldots, a^{n}(\sigma)\right\}$ so that the determinant of the invertible matrix

$$
A(\sigma)=\left\{a^{i}(\sigma)_{j}\right\}_{i, j=1}^{n} \in G L_{n}(\boldsymbol{Z})
$$

is 1 . For a cone $\sigma \in \Sigma$ and $1 \leq i \leq \operatorname{dim} \sigma$ we set

$$
l\left(a^{i}(\sigma)\right)=\min _{\alpha \in \Gamma_{+}(f)}\left\langle a^{i}(\sigma), \alpha\right\rangle \in \mathbf{Z}_{+}
$$

and

$$
\left|a^{i}(\sigma)\right|=\sum_{j=1}^{n} a^{i}(\sigma)_{j} \in \boldsymbol{Z}_{>0} .
$$

Now let $\sigma$ be an $n$-dimensional cone in $\Sigma$ and $\boldsymbol{R}^{n}(\sigma) \simeq \boldsymbol{R}_{y}^{n}$ the affine open subset of $X_{\Sigma}$ associated to $\sigma$. Then the restriction $\pi(\sigma): \boldsymbol{R}^{n}(\sigma) \longrightarrow \boldsymbol{R}^{n}$ of $\pi: X_{\Sigma} \longrightarrow \boldsymbol{R}^{n}$ to $\boldsymbol{R}^{n}(\sigma) \simeq$ $\boldsymbol{R}_{y}^{n}$ and its Jacobian $J(\pi(\sigma)): \boldsymbol{R}^{n}(\sigma) \longrightarrow \boldsymbol{R}$ are explicitly given by

$$
\begin{gathered}
\pi(\sigma)(y)=\left(\prod_{i=1}^{n} y_{i}^{a^{i}(\sigma)_{1}}, \prod_{i=1}^{n} y_{i}^{a^{i}(\sigma)_{2}}, \ldots, \prod_{i=1}^{n} y_{i}^{a^{i}(\sigma)_{n}}\right), \\
J(\pi(\sigma))(y)=y_{1}^{\left|a^{1}(\sigma)\right|-1} y_{2}^{\left|a^{2}(\sigma)\right|-1} \cdots y_{n}^{\left|a^{n}(\sigma)\right|-1} .
\end{gathered}
$$

Hence we can easily see that on $\boldsymbol{R}^{n}(\sigma) \simeq \boldsymbol{R}_{y}^{n}$ we have

$$
(f \circ \pi(\sigma))(y)=f_{\sigma}(y) \times \prod_{i=1}^{n} y_{i}^{l\left(a^{i}(\sigma)\right)},
$$

where $f_{\sigma}$ is a real analytic function defined on $\pi(\sigma)^{-1}(U) \subset \boldsymbol{R}^{n}(\sigma)$. By the non-degeneracy of $f$ the (smooth) hypersurface $\left\{f_{\sigma}=0\right\}$ intersects all coordinate subspaces of $\boldsymbol{R}^{n}(\sigma)$ transversally (see [1], [11], [18] and [22] etc.). In particular, we have $f_{\sigma}(0) \neq 0$. For $0 \leq k \leq n$ let $\Sigma^{(k)} \subset \Sigma$ be the subset of $\Sigma$ consisting of $k$-dimensional cones.

Definition 3.1 (see [1], [11] and [22] etc.). Let $P \subset \boldsymbol{Q}_{<0}$ be the union of the following subsets of $\boldsymbol{Q}_{<0}$ :

$$
\begin{gathered}
\{-1,-2,-3, \ldots\}, \\
\left\{-\frac{\left|a^{1}(\sigma)\right|}{l\left(a^{1}(\sigma)\right)},-\frac{\left|a^{1}(\sigma)\right|+1}{l\left(a^{1}(\sigma)\right)}, \ldots\right\} \quad\left(\sigma \in \Sigma^{(1)} \text { such that } l\left(a^{1}(\sigma)\right)>0\right) .
\end{gathered}
$$

By the fundamental result of [22], the poles of the local zeta function $Z_{f}(\varphi)$ are contained in the set $P$. An element of $P$ is called a candidate pole of $Z_{f}(\varphi)$. We order the candidate poles of $Z_{f}(\varphi)$ as

$$
P=\left\{-\lambda_{1}>-\lambda_{2}>-\lambda_{3}>\cdots\right\} \quad\left(\lambda_{j} \in Q_{>0}\right) .
$$

Hereafter we fix a candidate pole $-\lambda_{j} \in P$ of $Z_{f}(\varphi)$. Recall the definitions of $K^{i}(\sigma), \Sigma_{j}^{(k)}$ and $v(\sigma)_{i} \in \boldsymbol{Z}_{+}$in Section 1. Then, after [1] and [22] the following results are well known to the specialists. 
THEOREM 3.2. (i) Assume that $\lambda_{j} \notin Z$. Then the order of the pole of $Z_{f}(\varphi)$ at $\lambda=-\lambda_{j}$ is less than or equal to

$$
k_{j}:=\max \left\{0 \leq k \leq n ; \Sigma_{j}^{(k)} \neq \emptyset\right\} \in \boldsymbol{Z}_{+} .
$$

(ii) Assume that $\lambda_{j} \in Z$. Then the order of the pole of $Z_{f}(\varphi)$ at $\lambda=-\lambda_{j}$ is less than or equal to

$$
k_{j}:=1+\max \left\{0 \leq k \leq n ; \Sigma_{j}^{(k)} \neq \emptyset\right\} \in \boldsymbol{Z}_{+} .
$$

Now let

$$
\frac{a_{j, k_{j}}(\varphi)}{\left(\lambda+\lambda_{j}\right)^{k_{j}}}+\cdots+\frac{a_{j, 2}(\varphi)}{\left(\lambda+\lambda_{j}\right)^{2}}+\frac{a_{j, 1}(\varphi)}{\left(\lambda+\lambda_{j}\right)}+\cdots \quad\left(a_{j, k}(\varphi) \in \boldsymbol{R}\right)
$$

be the Laurent expansion of $Z_{f}(\varphi)$ at $\lambda=-\lambda_{j}$. Then we obtain the following result which generalizes Jacobs [10, Theorem 4.23].

THEOREM 3.3. (i) Assume that $\lambda_{j}$ is not an odd integer and let $1 \leq k \leq k_{j}$. Assume moreover that for any $\sigma \in \Sigma_{j}^{(k)}$ there exists $1 \leq i \leq k=\operatorname{dim} \sigma$ such that $v(\sigma)_{i}$ is odd. Then we have $a_{j, k}(\varphi)=\cdots=a_{j, k_{j}}(\varphi)=0$.

(ii) Assume that $\lambda_{j}$ is an odd integer and let $2 \leq k \leq k_{j}$. Assume moreover that for any $\sigma \in \Sigma_{j}^{(k-1)}$ there exists $1 \leq i \leq k-1=\operatorname{dim} \sigma$ such that $v(\sigma)_{i}$ is odd. Then we have $a_{j, k}(\varphi)=\cdots=a_{j, k_{j}}(\varphi)=0$.

PROOF. (i) Since supp $\varphi$ is compact and $\pi: X_{\Sigma} \longrightarrow \boldsymbol{R}^{n}$ is proper, there exists finite $C^{\infty}$-functions $\varphi_{q}(1 \leq q \leq N)$ on $X_{\Sigma}$ such that $\sum_{q=1}^{N} \varphi_{q} \equiv 1$ on $\operatorname{supp}(\varphi \circ \pi)$. We may assume that for any $1 \leq q \leq N$ there exists an $n$-dimensional cone $\sigma_{q} \in \Sigma^{(n)}$ such that $\operatorname{supp} \varphi_{q} \subset \subset \boldsymbol{R}^{n}\left(\sigma_{q}\right)$. Then we have

$$
Z_{f}(\varphi)(\lambda)=\sum_{q=1}^{N} \int_{\boldsymbol{R}^{n}\left(\sigma_{q}\right)}\left(\prod_{i=1}^{n}\left|y_{i}\right|^{l\left(a^{i}\left(\sigma_{q}\right)\right) \lambda+\left|a^{i}\left(\sigma_{q}\right)\right|-1}\right)\left|f_{\sigma_{q}}\right|^{\lambda}(y)\left(\varphi \circ \pi\left(\sigma_{q}\right)\right)(y) \varphi_{q}(y) d y .
$$

We divide the proof of the assertion (i) into the following two cases (I) and (II).

(I) First assume that $\lambda_{j}$ is not an integer. Then by (the proof of) Proposition $2.2, a_{j, k}(\varphi)$ can be written as

$$
a_{j, k}(\varphi)=\sum_{q=1}^{N} \sum_{p \geq k} \sum_{\sigma \in \Sigma_{j, q}^{(p)}} J_{q}(\sigma)
$$

where for $1 \leq q \leq N$ and $0 \leq p \leq n$ we set

$$
\Sigma_{j, q}^{(p)}=\left\{\sigma \in \Sigma_{j}^{(p)} ; \sigma \prec \sigma_{q}\right\} .
$$

Moreover for $p$ such that $k \leq p \leq n$ and $\sigma \in \Sigma_{j, q}^{(p)}$ the number $J_{q}(\sigma)$ is expressed as follows.

$$
J_{q}(\sigma)=\frac{1}{(p-k) !} \times\left.\frac{d^{p-k}}{d \lambda^{p-k}} \rho_{q, \sigma}(\lambda)\right|_{\lambda=-\lambda_{j}} .
$$


Let us explain the function $\rho_{q, \sigma}(\lambda)$ which is holomorphic at $\lambda=-\lambda_{j}$. For the sake of simplicity, we assume that $\left\{a^{1}\left(\sigma_{q}\right), a^{2}\left(\sigma_{q}\right), \ldots, a^{p}\left(\sigma_{q}\right)\right\}$ is the 1 -skeleton of $\sigma \prec \sigma_{q}$.

(a) (The case where $\operatorname{supp} \varphi_{q} \cap\left\{y \in \boldsymbol{R}^{n}\left(\sigma_{q}\right) ; f_{\sigma_{q}}(y)=y_{1}=y_{2}=\cdots=y_{p}=0\right\}=\emptyset$ ) We set

$$
\begin{aligned}
\rho_{q, \sigma}(\lambda)= & \left(\prod_{i=1}^{p} \frac{1+(-1)^{v\left(\sigma_{q}\right)_{i}}}{l\left(a^{i}\left(\sigma_{q}\right)\right) \cdot v\left(\sigma_{q}\right)_{i} !}\right) \int_{\left\{y_{1}=\cdots=y_{p}=0\right\}} \prod_{i=p+1}^{n} g_{i}\left(\lambda, y_{i}\right) \\
& \times \frac{\partial^{\nu\left(\sigma_{q}\right)_{1}+\cdots+v\left(\sigma_{q}\right)_{p}}}{\partial y_{1}^{v\left(\sigma_{q}\right)_{1}} \cdots \partial y_{p}^{\nu\left(\sigma_{q}\right)_{p}}}\left\{\left|f_{\sigma_{q}}\right|^{\lambda}\left(\varphi \circ \pi\left(\sigma_{q}\right)\right) \varphi_{q}\right\}_{y_{1}=\cdots=y_{p}=0} d y_{p+1} \cdots d y_{n},
\end{aligned}
$$

where $g_{p+1}(\lambda, \cdot), \ldots, g_{n}(\lambda, \cdot)$ are 1-dimensional integrable functions with holomorphic parameter $\lambda$ at $\lambda=-\lambda_{j} \in P$.

(b) (The case where $\operatorname{supp} \varphi_{q} \cap\left\{y \in \boldsymbol{R}^{n}\left(\sigma_{q}\right) ; f_{\sigma_{q}}(y)=y_{1}=y_{2}=\cdots=y_{p}=\right.$ $0\} \neq \emptyset)$ For $1 \leq i \leq n$ set $H_{i}=\left\{y \in \boldsymbol{R}^{n}\left(\sigma_{q}\right) ; y_{i}=0\right\}$. Then we may assume that $\left\{1 \leq i \leq n ; \operatorname{supp} \varphi_{q} \cap H_{i} \neq \emptyset\right\}=\{1,2, \ldots, r\}$ for some $r \in \boldsymbol{Z}$ such that $p \leq r \leq n-1$. In this case, by a real analytic local coordinate change $\Phi:\left(y_{1}, \ldots, y_{n}\right) \longmapsto\left(z_{1}, \ldots, z_{n}\right)$ such that $z_{i}=y_{i}(1 \leq i \leq r)$ which sends the hypersurface $\left\{f_{\sigma_{q}}=0\right\}$ to $\left\{z_{n}=0\right\}$, the function $\rho_{q, \sigma}(\lambda)$ is expressed as

$$
\begin{aligned}
& \rho_{q, \sigma}(\lambda)=\left(\prod_{i=1}^{p} \frac{1+(-1)^{v\left(\sigma_{q}\right)_{i}}}{l\left(a^{i}\left(\sigma_{q}\right)\right) \cdot v\left(\sigma_{q}\right)_{i} !}\right) \int_{\left\{z_{1}=\cdots=z_{p}=0\right\}}\left(\prod_{i=p+1}^{r} g_{i}\left(\lambda, z_{i}\right)\right) g_{n}\left(\lambda, z_{n}\right) \\
& \times \frac{\partial^{\nu\left(\sigma_{q}\right)_{1}+\cdots+v\left(\sigma_{q}\right)_{p}}}{\partial z_{1}^{\nu\left(\sigma_{q}\right)_{1}} \cdots \partial z_{p}^{v\left(\sigma_{q}\right)_{p}}}\left\{F(\lambda, z)\left(\varphi \circ \pi\left(\sigma_{q}\right) \circ \Phi^{-1}\right)\left(\varphi_{q} \circ \Phi^{-1}\right)\left|\frac{\partial\left(y_{1}, \ldots, y_{n}\right)}{\partial\left(z_{1}, \ldots, z_{n}\right)}\right|\right\}_{z_{1}=\cdots=z_{p}=0} \\
& d z_{p+1} \cdots d z_{n},
\end{aligned}
$$

where

$$
F(\lambda, z)=\left(\prod_{i=r+1}^{n}\left|y_{i}\right|^{l\left(a^{i}\left(\sigma_{q}\right)\right) \lambda+\left|a^{i}\left(\sigma_{q}\right)\right|-1}\right) \circ \Phi^{-1}
$$

is a real analytic function of $z$ on a neighborhood of $\Phi\left(\operatorname{supp} \varphi_{q}\right)$ and $g_{p+1}(\lambda, \cdot), \ldots, g_{r}(\lambda, \cdot)$ and $g_{n}(\lambda, \cdot)$ are 1-dimensional integrable functions with holomorphic parameter $\lambda$ at $\lambda=$ $-\lambda_{j} \in P$. To ensure the existence of such $g_{n}(\lambda, \cdot)$ we used the condition that $\lambda_{j}$ is not an integer.

Now by our assumption, for any $\sigma \in \Sigma_{j, q}^{(p)}(p \geq k)$ there exists $1 \leq i \leq p$ such that $v\left(\sigma_{q}\right)_{i}$ is odd. Therefore we obtain $a_{j, k}(\varphi)=0$ in this case. In the same way, we can prove also that $a_{j, k+1}(\varphi)=\cdots=a_{j, k_{j}}(\varphi)=0$.

(II) Next assume that $\lambda_{j}$ is an (even) integer and set $m:=\lambda_{j}$. Then by (the proof of) Proposition 2.2, $a_{j, k}(\varphi)$ can be written as

$$
\sum_{q=1}^{N}\left\{\sum_{p \geq k} \sum_{\sigma \in \Sigma_{j, q}^{(p)}} J_{q}(\sigma)+\sum_{p \geq k-1} \sum_{\sigma \in \Sigma_{j, q}^{(p)}} \tilde{J}_{q}(\sigma)\right\},
$$


where $J_{q}(\sigma)$ is as in (I) and for $p$ such that $k-1 \leq p \leq n$ and $\sigma \in \Sigma_{j, q}^{(p)}$ the number $\tilde{J}_{q}(\sigma)$ is expressed as follows.

$$
\tilde{J}_{q}(\sigma)=\frac{1}{(p+1-k) !} \times\left.\frac{d^{p+1-k}}{d \lambda^{p+1-k}} \tau_{q, \sigma}(\lambda)\right|_{\lambda=-\lambda_{j}} .
$$

Let us explain the function $\tau_{q, \sigma}(\lambda)$ which is holomorphic at $\lambda=-\lambda_{j}$. For simplicity, we assume that $\left\{a^{1}\left(\sigma_{q}\right), a^{2}\left(\sigma_{q}\right), \ldots, a^{p}\left(\sigma_{q}\right)\right\}$ is the 1-skeleton of $\sigma \prec \sigma_{q}$. If $\operatorname{supp} \varphi_{q} \cap\{y \in$ $\left.\boldsymbol{R}^{n}\left(\sigma_{q}\right) ; f_{\sigma_{q}}(y)=y_{1}=\cdots=y_{p}=0\right\}=\emptyset$ we set $\tau_{q, \sigma}(\lambda) \equiv 0$. If $\operatorname{supp} \varphi_{q} \cap\{y \in$ $\left.\boldsymbol{R}^{n}\left(\sigma_{q}\right) ; f_{\sigma_{q}}(y)=y_{1}=\cdots=y_{p}=0\right\} \neq \emptyset$, assuming as in (I)(b) that $\{1 \leq i \leq$ $n$; $\left.\operatorname{supp} \varphi_{q} \cap H_{i} \neq \emptyset\right\}=\{1,2, \ldots, r\}$ for some $r \in \boldsymbol{Z}$ such that $p \leq r \leq n-1$ and using the local coordinate change $\Phi$ used in (I)(b), the function $\tau_{q, \sigma}(\lambda)$ is expressed as

$$
\begin{aligned}
\tau_{q, \sigma}(\lambda)= & \left(\prod_{i=1}^{p} \frac{1+(-1)^{v\left(\sigma_{q}\right)_{i}}}{l\left(a^{i}\left(\sigma_{q}\right)\right) \cdot v\left(\sigma_{q}\right)_{i} !}\right) \times \frac{1+(-1)^{m-1}}{(m-1) !} \\
& \times \int_{\left\{z_{1}=\cdots=z_{p}=z_{n}=0\right\}}\left(\prod_{i=p+1}^{r} g_{i}\left(\lambda, z_{i}\right)\right) K\left(\lambda, z_{p+1}, \ldots, z_{n-1}\right) d z_{p+1} \cdots d z_{n-1},
\end{aligned}
$$

where $K\left(\lambda, z_{p+1}, \ldots, z_{n-1}\right)$ is the function

$$
\begin{aligned}
\frac{\partial^{v\left(\sigma_{q}\right)_{1}+\cdots+v\left(\sigma_{q}\right)_{p}+m-1}}{\partial z_{1}^{v\left(\sigma_{q}\right)_{1}} \cdots \partial z_{p}^{\nu\left(\sigma_{q}\right)_{p}} \partial z_{n}^{m-1}}\left\{F(\lambda, z)\left(\varphi \circ \pi\left(\sigma_{q}\right) \circ \Phi^{-1}\right)\left(\varphi_{q} \circ \Phi^{-1}\right)\right. \\
\left.\times\left|\frac{\partial\left(y_{1}, \ldots, y_{n}\right)}{\partial\left(z_{1}, \ldots, z_{n}\right)}\right|\right\}_{z_{1}=\cdots=z_{p}=z_{n}=0}
\end{aligned}
$$

obtained by taking $F(\lambda, z)$ and $g_{p+1}(\lambda, \cdot), \ldots, g_{r}(\lambda, \cdot)$ as in (I)(b). Then, as in (I), by our assumption we obatin $J_{q}(\sigma)=0$ for any $\sigma \in \Sigma_{j, q}^{(p)}(p \geq k)$. Moreover, since by our assumption in (i) the integer $m=\lambda_{j}$ must be even, we obtain $\tilde{J}_{q}(\sigma)=0$ for any $\sigma \in \Sigma_{j, q}^{(p)}$ $(p \geq k-1)$. Therefore we get $a_{j, k}(\varphi)=0$ in this case, too. In the same way, we can prove also that $a_{j, k+1}(\varphi)=\cdots=a_{j, k_{j}}(\varphi)=0$. This completes the proof of (i). The remaining assertion (ii) can be shown similarly.

By this theorem we see that many candidate poles of $Z_{f}(\varphi)$ are fake, i.e., not the actual poles. We can also find a nice condition on the test function $\varphi \in C_{0}^{\infty}\left(\boldsymbol{R}^{n}\right)$ under which we have the vanishing $a_{j, k}(\varphi)=\cdots=a_{j, k_{j}}(\varphi)=0$. For this purpose, we introduce the following subset $\Delta_{j, k}$ of $\boldsymbol{R}_{+}^{n}$.

DEFINITION 3.4. Let $1 \leq k \leq k_{j}$.

(i) For $\sigma \in \Sigma_{j}^{(k)}$ we define a compact convex subset $\Delta_{j, k}^{\sigma}$ of $\boldsymbol{R}_{+}^{n}$ by

$$
\Delta_{j, k}^{\sigma}=\left\{\alpha \in \boldsymbol{R}_{+}^{n} ;\left\langle a^{i}(\sigma), \alpha\right\rangle \leq v(\sigma)_{i} \quad \text { for any } 1 \leq i \leq k\right\} .
$$


(ii) We define a compact subset $\Delta_{j, k}$ of $\boldsymbol{R}_{+}^{n}$ by

$$
\Delta_{j, k}=\bigcup_{\sigma \in \Sigma_{j}^{(k)}} \Delta_{j, k}^{\sigma} .
$$

Note that $\Delta_{j, k}$ is not necessarily a convex subset of $\boldsymbol{R}_{+}^{n}$. It follows also from the definition that we have $\Delta_{j, k} \supset \Delta_{j, k^{\prime}}$ for $1 \leq k \leq k^{\prime} \leq k_{j}$. In order to state our another vanishing theorem, let

$$
\varphi(x)=\sum_{\alpha \in \mathbf{Z}_{+}^{n}} c_{\alpha} x^{\alpha} \quad\left(c_{\alpha}=\frac{\partial_{x}^{\alpha} \varphi(0)}{\alpha !} \in \boldsymbol{R}\right)
$$

be the (formal) Taylor expansion of the test function $\varphi$ at $0 \in U \subset \boldsymbol{R}^{n}$.

THEOREM 3.5. (i) Let $1 \leq k \leq k_{j}$. Assume that $\lambda_{j}$ is not an odd integer and $\left\{\alpha \in \boldsymbol{Z}_{+}^{n} ; c_{\alpha} \neq 0\right\} \cap \Delta_{j, k}=\emptyset$. Then we have $a_{j, k}(\varphi)=\cdots=a_{j, k_{j}}(\varphi)=0$.

(ii) Let $2 \leq k \leq k_{j}$. Assume that $\lambda_{j}$ is an odd integer and $\left\{\alpha \in \boldsymbol{Z}_{+}^{n} ; c_{\alpha} \neq 0\right\} \cap$ $\Delta_{j, k-1}=\emptyset$. Then we have $a_{j, k}(\varphi)=\cdots=a_{j, k_{j}}(\varphi)=0$.

Proof. We use the notations in the proof of Theorem 3.3.

(i) Since the proof for the case where $\lambda_{j}$ is an (even) integer is similar, we prove the assertion only in the case where $\lambda_{j}$ is not an integer. In this case, we have

$$
a_{j, k}(\varphi)=\left.\sum_{q=1}^{N} \sum_{p \geq k} \sum_{\sigma \in \Sigma_{j, q}^{(p)}} \frac{1}{(p-k) !} \frac{d^{p-k}}{d \lambda^{p-k}} \rho_{q, \sigma}(\lambda)\right|_{\lambda=-\lambda_{j}},
$$

where the function $\rho_{q, \sigma}(\lambda)$ is holomorphic at $\lambda=-\lambda_{j}$ (for its expression, see the proof of Theorem 3.3). For $\alpha \in \boldsymbol{Z}_{+}^{n}$ let $\psi_{\alpha} \in C_{0}^{\infty}(U)$ be a test function on $U$ such that $\psi_{\alpha} \equiv x^{\alpha}$ in a neighborhood of $0 \in U \subset \boldsymbol{R}^{n}$. For $1 \leq q \leq N$ and $\sigma \in \Sigma_{j, q}^{(p)}(p \geq k)$, assume for simplicity that $\left\{a^{1}\left(\sigma_{q}\right), a^{2}\left(\sigma_{q}\right), \ldots, a^{p}\left(\sigma_{q}\right)\right\}$ is the 1-skeleton of $\sigma \prec \sigma_{q}$. Then in an open neighborhood of $\left\{y \in \boldsymbol{R}^{n}\left(\sigma_{q}\right) ; y_{1}=\cdots=y_{p}=0\right\} \subset \boldsymbol{R}^{n}\left(\sigma_{q}\right) \subset X_{\Sigma}$ we have

$$
\left(\psi_{\alpha} \circ \pi\left(\sigma_{q}\right)\right)(y) \equiv y_{1}^{\left\langle a^{1}\left(\sigma_{q}\right), \alpha\right\rangle} \cdots y_{n}^{\left\langle a^{n}\left(\sigma_{q}\right), \alpha\right\rangle} .
$$

Moreover, by the definition of $\Delta_{j, k}$, if $\alpha \notin \Delta_{j, k}$ then we have $\alpha \notin \Delta_{j, p}$ and there exists $1 \leq i \leq p$ such that $\left\langle a^{i}\left(\sigma_{q}\right), \alpha\right\rangle>v\left(\sigma_{q}\right)_{i}$. This implies the vanishing of the function

$$
\frac{\partial^{v\left(\sigma_{q}\right)_{1}+\cdots+v\left(\sigma_{q}\right)_{p}}}{\partial y_{1}^{v\left(\sigma_{q}\right)_{1}} \cdots \partial y_{p}^{\nu\left(\sigma_{q}\right)_{p}}}\left\{\left|f_{\sigma_{q}}\right|^{\lambda}\left(\psi_{\alpha} \circ \pi\left(\sigma_{q}\right)\right) \varphi_{q}\right\}_{y_{1}=\cdots=y_{p}=0} \equiv 0 .
$$

By applying this vanishing result to the expressions of $\rho_{q, \sigma}(\lambda)$ in (3.19) and (3.20), we obtain $a_{j, k}\left(\psi_{\alpha}\right)=0$ for $\alpha \notin \Delta_{j, k}$. Moreover by using the Taylor expansion of $\varphi$, under our assumption $\left\{\alpha \in \boldsymbol{Z}_{+}^{n} ; c_{\alpha} \neq 0\right\} \cap \Delta_{j, k}=\emptyset$ we can easily prove that $a_{j, k}(\varphi)=0$. In the same way, we can prove also that $a_{j, k+1}(\varphi)=\cdots=a_{j, k_{j}}(\varphi)=0$. This completes the proof of (i). The assertion (ii) can be shown similarly. 
Now let us consider the following two local zeta functions.

$$
Z_{f}^{ \pm}(\varphi)(\lambda)=\int_{\boldsymbol{R}^{n}} f_{ \pm}^{\lambda}(x) \varphi(x) d x .
$$

Note that we have $Z_{f}(\varphi)=Z_{f}^{+}(\varphi)+Z_{f}^{-}(\varphi)$. Then the poles of these lcoal zeta functions $Z_{f}^{ \pm}(\varphi)$ are also contained in $P$ and their Laurent expansions at a candidate pole $\lambda=-\lambda_{j} \in P$ have the following form:

$$
\frac{a_{j, k_{j}}^{ \pm}(\varphi)}{\left(\lambda+\lambda_{j}\right)^{k_{j}}}+\cdots+\frac{a_{j, 2}^{ \pm}(\varphi)}{\left(\lambda+\lambda_{j}\right)^{2}}+\frac{a_{j, 1}^{ \pm}(\varphi)}{\left(\lambda+\lambda_{j}\right)}+\cdots \quad\left(a_{j, k}^{ \pm}(\varphi) \in \boldsymbol{R}\right)
$$

(see for example [1], [11] etc.). By the proof of Theorem 3.5 we obtain a vanishing theorem also for the coefficients $a_{j, k}^{ \pm}(\varphi)$ of the poles of $Z_{f}^{ \pm}(\varphi)$.

THEOREM 3.6. (i) Let $1 \leq k \leq k_{j}$. Assume that $\lambda_{j}$ is not an integer and $\{\alpha \in$ $\left.\boldsymbol{Z}_{+}^{n} ; c_{\alpha} \neq 0\right\} \cap \Delta_{j, k}=\emptyset$. Then we have $a_{j, k}^{ \pm}(\varphi)=\cdots=a_{j, k_{j}}^{ \pm}(\varphi)=0$.

(ii) Let $2 \leq k \leq k_{j}$. Assume that $\lambda_{j}$ is an integer and $\left\{\alpha \in \boldsymbol{Z}_{+}^{n} ; c_{\alpha} \neq 0\right\} \cap \Delta_{j, k-1}=\emptyset$. Then we have $a_{j, k}^{ \pm}(\varphi)=\cdots=a_{j, k_{j}}^{ \pm}(\varphi)=0$.

REMARK 3.7. By replacing $\Sigma$ by $\Sigma_{0}$ we can also define compact subsets $\Delta_{j, k}^{\prime}$ of $\boldsymbol{R}_{+}^{n}$. For these subsets $\Delta_{j, k}^{\prime}$ we obtain the analogues of Theorems 3.5 and 3.6 by using the arguments in Denef-Sargos [4]. However we cannot similarly obtain the analogues of Theorems 3.3 and 4.2 etc., which are more directly related to the dual fan $\Sigma_{0}$ of $\Gamma_{+}(f)$, by some technical reason.

4. Explicit formulas for the poles of local zeta functions. In this section we give some explicit formulas for the coefficients $a_{j, n}(\varphi), a_{j, n}^{ \pm}(\varphi)$ of the deepest poles $\lambda=-\lambda_{j} \in P$ of the local zeta functions $Z_{f}(\varphi), Z_{f}^{ \pm}(\varphi)$ introduced in Section 3 . We inherit the situation and the notations in Section 3. Let $-\lambda_{j} \in P$ be a candidate pole of $Z_{f}(\varphi)$.

Definition 4.1. For $\sigma \in \Sigma_{j}^{(n)}$ and $\alpha \in Z_{+}^{n}$ we define an integer $\mu(\sigma, \alpha)_{i}$ by

$$
\mu(\sigma, \alpha)_{i}=v(\sigma)_{i}-\left\langle a^{i}(\sigma), \alpha\right\rangle \in \boldsymbol{Z} .
$$

THEOREM 4.2. Assume that $\lambda_{j}$ is not an odd integer and $k_{j}=n$. Then the coefficient $a_{j, n}(\varphi)$ of the deepest possible pole $\lambda=-\lambda_{j} \in P$ of $Z_{f}(\varphi)$ is given by

$$
a_{j, n}(\varphi)=\sum_{\alpha \in \Delta_{j, n}}\left\{\sum_{\sigma \in \Sigma_{j}^{(n)}}\left(\prod_{i=1}^{n} \frac{1+(-1)^{v(\sigma)_{i}}}{l\left(a^{i}(\sigma)\right) \cdot \mu(\sigma, \alpha)_{i} !}\right) \partial_{y}^{\mu(\sigma, \alpha)}\left|f_{\sigma}\right|^{-\lambda_{j}}(0)\right\} \frac{\partial_{x}^{\alpha} \varphi(0)}{\alpha !}
$$

where we set

$$
\partial_{y}^{\mu(\sigma, \alpha)}=\frac{\partial^{\mu(\sigma, \alpha)_{1}+\cdots+\mu(\sigma, \alpha)_{n}}}{\partial y_{1}^{\mu(\sigma, \alpha)_{1}} \cdots \partial y_{n}^{\mu(\sigma, \alpha)_{n}}}
$$

and $\left(\partial^{\mu} / \partial y_{i}^{\mu}\right)(\cdot)=0$ if $\mu<0$. 
PROOF. Since $\lambda_{j}$ is not an odd integer, by the proof of Theorem 3.3 we have

$$
a_{j, n}(\varphi)=\sum_{\sigma \in \Sigma_{j}^{(n)}}\left(\prod_{i=1}^{n} \frac{1+(-1)^{\nu(\sigma)_{i}}}{l\left(a^{i}(\sigma)\right) \cdot v(\sigma)_{i} !}\right) \partial_{y}^{\nu(\sigma)}\left\{\left|f_{\sigma}\right|^{-\lambda_{j}}(\varphi \circ \pi(\sigma))\right\}_{y=0} .
$$

Now let

$$
\varphi(x)=\sum_{\alpha \in \mathbf{Z}_{+}^{n}} \frac{\partial_{x}^{\alpha} \varphi(0)}{\alpha !} x^{\alpha}
$$

be the (formal) Taylor expansion of $\varphi$ at $0 \in \boldsymbol{R}^{n}$. Since $a_{j, n}\left(x^{\alpha}\right)=0$ for $\alpha \notin \Delta_{j, n}$ by Theorem 3.5 and for any $\sigma \in \Sigma_{j}^{(n)}$ and $\alpha \in \Delta_{j, k}$ we have

$$
\left(x^{\alpha} \circ \pi(\sigma)\right)(y) \equiv y_{1}^{\left\langle a^{1}(\sigma), \alpha\right\rangle} \cdots y_{n}^{\left\langle a^{n}(\sigma), \alpha\right\rangle}
$$

in a neighborhood of $0 \in \boldsymbol{R}^{n}(\sigma)$, we obtain

$$
\begin{aligned}
& a_{j, n}(\varphi) \\
& =\sum_{\alpha \in \Delta_{j, n}}\left\{\sum_{\sigma \in \Sigma_{j}^{(n)}}\left(\prod_{i=1}^{n} \frac{1+(-1)^{v(\sigma)_{i}}}{l\left(a^{i}(\sigma)\right) \cdot v(\sigma)_{i} !}\right) \partial_{y}^{v(\sigma)}\left(\left|f_{\sigma}\right|^{-\lambda_{j}} y_{1}^{\left\langle a^{1}(\sigma), \alpha\right\rangle} \cdots y_{n}^{\left\langle a^{n}(\sigma), \alpha\right\rangle}\right)_{y=0}\right\} \\
& \quad \times \frac{\partial_{x}^{\alpha} \varphi(0)}{\alpha !} .
\end{aligned}
$$

Then the result follows from the Leibniz rule. This completes the proof.

In order to state similar results for $a_{j, n}^{ \pm}(\varphi)$ we define two integers $c_{ \pm}(\sigma)\left(\sigma \in \Sigma_{j}^{(n)}\right)$ as follows. First set $\{ \pm 1\}^{n}:=\left\{\varepsilon=\left(\varepsilon_{1}, \varepsilon_{2}, \ldots, \varepsilon_{n}\right) ; \varepsilon_{i}= \pm 1\right\}$. For $\sigma \in \Sigma_{j}^{(n)}$ we define subsets $Q_{ \pm}(\sigma)$ of $\{ \pm 1\}^{n}$ by

$$
Q_{ \pm}(\sigma)=\left\{\varepsilon=\left(\varepsilon_{1}, \varepsilon_{2}, \ldots, \varepsilon_{n}\right) ; \pm f_{\sigma}(0) \times \prod_{i=1}^{n} \varepsilon_{i}^{l\left(a^{i}(\sigma)\right)}>0\right\} .
$$

Let us explain the meaning of $Q_{ \pm}(\sigma) \subset\{ \pm 1\}^{n}$. For each $\varepsilon=\left(\varepsilon_{1}, \ldots, \varepsilon_{n}\right) \in\{ \pm 1\}^{n}$ we define an open subset $V_{\varepsilon}$ of $\boldsymbol{R}^{n}(\sigma) \simeq \boldsymbol{R}_{y}^{n}$ by

$$
V_{\varepsilon}=\left\{\left(y_{1}, y_{2}, \ldots, y_{n}\right) \in \boldsymbol{R}^{n}(\sigma) ; \varepsilon_{i} y_{i}>0 \text { for any } 1 \leq i \leq n\right\} .
$$

Then there exists a sufficiently small open neighborhood $W$ of $0 \in \boldsymbol{R}^{n}(\sigma)$ such that $\pm\left.(f \circ \pi(\sigma))\right|_{W \cap V_{\varepsilon}}>0$ for any $\varepsilon \in Q_{ \pm}(\sigma)$. Namely $Q_{ \pm}(\sigma)$ is naturally identified with the set $\left\{V_{\varepsilon}\right\}_{\varepsilon \in Q_{ \pm}(\sigma)}$ of open quadrants in $\boldsymbol{R}^{n}(\sigma) \simeq \boldsymbol{R}_{y}^{n}$ such that $\pm\left.(f \circ \pi(\sigma))\right|_{W \cap V_{\varepsilon}}>0$ for a small neighborhood $W$ of $0 \in \boldsymbol{R}^{n}(\sigma)$.

DEFinition 4.3. For $\sigma \in \Sigma_{j}^{(n)}$ we set

$$
c_{ \pm}(\sigma)=\sum_{\varepsilon \in Q_{ \pm}(\sigma)}\left(\prod_{i=1}^{n} \varepsilon_{i}^{\nu(\sigma)_{i}}\right) \in \boldsymbol{Z} .
$$


Note that for any $\sigma \in \Sigma_{j}^{(n)}$ we have

$$
c_{+}(\sigma)+c_{-}(\sigma)=\prod_{i=1}^{n}\left\{1+(-1)^{v(\sigma)_{i}}\right\} .
$$

THEOREM 4.4. Assume that $\lambda_{j}$ is not an integer and $k_{j}=n$. Then the coefficient $a_{j, n}^{ \pm}(\varphi)$ of the deepest possible pole $\lambda=-\lambda_{j} \in P$ of $Z_{f}^{ \pm}(\varphi)$ is given by

$$
\begin{aligned}
& a_{j, n}^{ \pm}(\varphi) \\
& \quad=\sum_{\alpha \in \Delta_{j, n}}\left\{\sum_{\sigma \in \Sigma_{j}^{(n)}} c_{ \pm}(\sigma)\left(\prod_{i=1}^{n} \frac{1}{l\left(a^{i}(\sigma)\right) \cdot \mu(\sigma, \alpha)_{i} !}\right) \partial_{y}^{\mu(\sigma, \alpha)}\left|f_{\sigma}\right|^{-\lambda_{j}}(0)\right\} \frac{\partial_{x}^{\alpha} \varphi(0)}{\alpha !},
\end{aligned}
$$

where for $\mu<0$ we set $\left(\partial^{\mu} / \partial y_{i}^{\mu}\right)(\cdot)=0$.

5. Asymptotic expansions of oscillating integrals. In this section, combining our previous arguments with the basic results in [1] and [22], we obtain some results on the asymptotic expansions of oscillating integrals. As before, let $f$ be a real-valued real analytic function defined on an open neighborhood $U$ of $0 \in \boldsymbol{R}^{n}$ such that $f(0)=0$ and $\varphi \in C_{0}^{\infty}(U)$ a real-valued test function defined on $U$. Then the oscillating integral $I_{f}(\varphi)(t)(t \in \boldsymbol{R})$ associated to $f$ and $\varphi$ is defined by

$$
I_{f}(\varphi)(t)=\int_{\boldsymbol{R}^{n}} e^{i t f(x)} \varphi(x) d x .
$$

Here we set $i=\sqrt{-1}$ for short. From now on, we assume the situation in Sections 3 and 4 and use the notations there. Then by the fundamental results of Varchenko [22] (see also [1] and [11] for the detail), as $t \rightarrow+\infty$ the oscillating integral $I_{f}(\varphi)(t)$ has an asymptotic expansion of the form

$$
I_{f}(\varphi)(t) \sim \sum_{j=1}^{\infty} \sum_{k=1}^{k_{j}} c_{j, k}(\varphi) t^{-\lambda_{j}}(\log t)^{k-1},
$$

where $c_{j, k}(\varphi)$ are some complex numbers. Despite the important contributions by many mathematicians (see for example [1], [3], [8] and [20] etc.), only little is known about the coefficients $c_{j, k}(\varphi)$ of the asymptotic expansion. First of all, we shall give a general vanishing theorem for these coefficients $c_{j, k}(\varphi)$. Let us fix a candidate pole $-\lambda_{j} \in P$ of the local zeta function $Z_{f}(\varphi)$ and let

$$
\varphi(x)=\sum_{\alpha \in \boldsymbol{Z}_{+}^{n}} c_{\alpha} x^{\alpha} \quad\left(c_{\alpha}=\frac{\partial_{x}^{\alpha} \varphi(0)}{\alpha !} \in \boldsymbol{R}\right)
$$

be the (formal) Taylor expansion of the test function $\varphi$ at $0 \in U \subset \boldsymbol{R}^{n}$.

THEOREM 5.1. (i) Let $1 \leq k \leq k_{j}$. Assume that $\lambda_{j}$ is not an integer and $\{\alpha \in$ $\left.Z_{+}^{n} ; c_{\alpha} \neq 0\right\} \cap \Delta_{j, k}=\emptyset$. Then we have $c_{j, k}(\varphi)=\cdots=c_{j, k_{j}}(\varphi)=0$. 
(ii) Let $2 \leq k \leq k_{j}$. Assume that $\lambda_{j}$ is an integer and $\left\{\alpha \in Z_{+}^{n} ; c_{\alpha} \neq 0\right\} \cap \Delta_{j, k-1}=\emptyset$. Then we have $c_{j, k}(\varphi)=\cdots=c_{j, k_{j}}(\varphi)=0$.

PROOF. By the results of [1] and [22], $c_{j, l}(\varphi)$ are linear combinations of $a_{j, m}^{ \pm}(\varphi)(m \geq$ l). Then the assertion follows immediately from Theorem 3.6.

Next we give an explicit formula for the coefficients $c_{j, n}(\varphi)$ of $t^{-\lambda_{j}}(\log t)^{n-1}$ in the asymptotic expansion (5.2). For this purpose, we define two real numbers $b_{j, n}^{ \pm}(\varphi) \in \boldsymbol{R}$ by

$$
\begin{aligned}
& b_{j, n}^{ \pm}(\varphi) \\
& \quad=\sum_{\alpha \in \Delta_{j, n}}\left\{\sum_{\sigma \in \Sigma_{j}^{(n)}} c_{ \pm}(\sigma)\left(\prod_{i=1}^{n} \frac{1}{l\left(a^{i}(\sigma)\right) \cdot \mu(\sigma, \alpha)_{i} !}\right) \partial_{y}^{\mu(\sigma, \alpha)}\left|f_{\sigma}\right|^{-\lambda_{j}}(0)\right\} \frac{\partial_{x}^{\alpha} \varphi(0)}{\alpha !},
\end{aligned}
$$

where for $\mu<0$ we set $\left(\partial^{\mu} / \partial y_{i}^{\mu}\right)(\cdot)=0$. Recall that if $\lambda_{j}$ is not an integer we have $a_{j, n}^{ \pm}(\varphi)=$ $b_{j, n}^{ \pm}(\varphi)$.

THEOREM 5.2. The coefficient $c_{j, n}(\varphi)$ of $t^{-\lambda_{j}}(\log t)^{n-1}$ in the asymptotic expansion (5.2) of $I_{f}(\varphi)$ is given by

$$
c_{j, n}(\varphi)=\frac{\Gamma\left(\lambda_{j}\right)}{(n-1) !}\left\{e^{\left(\pi i \lambda_{j}\right) / 2} \cdot b_{j, n}^{+}(\varphi)+e^{-\left(\pi i \lambda_{j}\right) / 2} \cdot b_{j, n}^{-}(\varphi)\right\} .
$$

Proof. We use the notations in the proof of Theorem 3.3. By the well-known arguments in [11] and [22] etc. we do not have to consider the contributions from $\left\{f_{\sigma_{q}}=0\right\}$ $(q=1,2, \ldots, N)$. Then the result follows from (the proof of) Theorems 4.2 and 4.4 .

\section{REFERENCES}

[ 1 ] V. I. Arnold, S. M. Gusein-Zade And A. N. Varchenko, Singularities of differential maps, Volume II, Birkhäuser, 1988.

[ 2 ] D. BARLET, Développement asymptotique des fonctions obtenues par intégration sur les fibres, Invent. Math. 68 (1982), 129-174.

[ 3 ] J. DENEF, J. NiCAise AND P. SARGos, Oscillating integrals and Newton polyhedra, J. Anal. Math. 95 (2005), 147-172.

[4] J. Denef And P. SARgos, Polyèdre de Newton et distribution $f_{+}^{s}$ I, J. Anal. Math. 53 (1989), 201-218.

[ 5 ] W. Fulton, Introduction to toric varieties, Ann. of Math. Stud. 131, The William H. Roever Lectures in Geometry, Princeton University Press, Princeton, NJ, 1993.

[ 6 ] I. M. Gelfand And G. E. Shilov, Generalized functions- properties and operations, Volume I, Academic Press, 1964.

[ 7 ] A. Greenleaf, M. Pramanik And W. TANG, Oscillatory integral operators with homogeneous polynomial phases in several variables, J. Funct. Anal. 224 (2007), 444-487.

[ 8 ] A. Greenleaf AND A. SeEger, Oscillatory integral operators with low-order degeneracies, Duke Math. J. 112 (2002), 397-420.

[9] J. IgUSA, An introduction to the theory of local zeta functions, AMS/IP Stud. Adv. Math. 14, American Mathematical Society, Providence, RI; International Press, Cambridge, MA, 2000.

[10] P. JACOBS, The distribution $|f|^{\lambda}$, oscillating integrals and principal value integrals, J. Anal. Math. 81 (2000), 343-372. 
[11] A. KANEKo, Newton diagrams, singular points and oscillating integrals, in Japanese, Lecture notes in Sophia university, No. 11, 1981.

[12] T. KimuRA, Introduction to prehomogeneous vector spaces, Transl. Math. Monogr. 215, American Mathematical Society, Providence, RI, 2003.

[13] Y. Matsui AND K. TAKeUChi, A geometric degree formula for $A$-discriminants and Euler obstructions of toric varieties, Adv. Math. 226 (2011), 2040-2064.

[14] Y. MATSUi AND K. TAKEUCHI, Milnor fibers over singular toric varieties and nearby cycle sheaves, Tohoku Math. J. 63 (2011), 113-136.

[15] Y. Matsui And K. TAKeuchi, Monodromy at infinity, Newton polyhedra and constructible sheaves, Math. Z. 268 (2011), 409-439.

[16] J. NicAise, An introduction to $p$-adic and motivic zeta functions and the monodromy conjecture, Algebraic and analytic aspects of zeta functions and L-functions, 141-166, MSJ Mem. 21, Math. Soc. Japan, Tokyo, 2010.

[17] T. ODA, Convex bodies and algebraic geometry. An introduction to the theory of toric varieties, Ergeb. Math. Grenzgeb. (3) 15, Springer-Verlag, Berlin, 1988.

[18] M. OKA, Non-degenerate complete intersection singularity, Hermann, Paris, 1997.

[19] M. Sato, T. KaWAi And M. Kashiwara, Microfunctions and pseudo-differential equations, Hyperfunctions and pseudo-differential equations (Proc. Conf., Katata, 1971; dedicated to the memory of André Martineau), pp. 265-529, Lecture Notes in Math. 287, Springer, Berlin, 1973.

[20] H. SogA, Conditions against rapid decrease of oscillatory integrals and their applications to inverse scattering problems, Osaka J. Math. 23 (1986), 441-456.

[21] K. TAKEUCHI, Monodromy at infinity of $A$-hypergeometric functions and toric compactifications, Math. Ann. 348 (2010), 815-831.

[22] A. N. VArchenko, Newton polyhedra and estimation of oscillating integrals, Funct. Anal. Appl. 10 (1977), 175-196.

IBARAKI PREFECTURAL

KOGA FIRST SENIOR HIGH SCHOOL

JAPAN

E-mail address: okada.tosihisa@post.ibk.ed.jp
INSTITUTE OF MATHEMATICS

UNIVERSITY OF TSUKUBA

1-1-1, TENNODAI, TSUKUBA

IBARAKI, 305-8571

JAPAN

E-mail address: takemicro@nifty.com 